\title{
The ASTRI-Horn telescope: comparison with the auxiliary UVscope measurements as calibration tool.
}

\section{A. Compagnino, ${ }^{a, *}$ T. Mineo, ${ }^{a}$ M. C. Maccarone, ${ }^{a}$ O. Catalano, ${ }^{a}$ D. Impiombato, ${ }^{a}$ and S. Giarrusso $^{a}$ for the ASTRI Project}

${ }^{a}$ INAF, Istituto di Astrofisica Spaziale e Fisica Cosmica di Palermo, via U. La Malfa 153, Palermo, Italy

E-mail: antonio.compagnino@inaf.it, teresa.mineo@inaf.it

\begin{abstract}
ASTRI-Horn is an Image Atmospheric Cherenkov Telescope located at the INAF "M.C. Fracastoro" observing station (Mt. Etna, Italy) characterized by a dual-mirror optical system and a curved focal plane equipped with SiPM sensors read by an innovative fast front-end electronics based on the peak detector technique. ASTRI-Horn represents the prototype of nine similar telescopes developed for the ASTRI-MiniArray that will be installed at the Teide Astronomical Observatory, in Tenerife (Canary Islands, Spain). The ASTRI-Horn camera is almost blind to the diffuse night sky background (NSB) but is able to detect the (Poissonian) fluctuations produced by the NSB. The noise generated by this effect is proportional to the level of the NSB. In this contribution, we present the analysis of the background data collected with the ASTRI-Horn camera during the period December 2018 March 2019. Moreover we report the comparison of the absolute night sky background measured by the ASTRI-Horn camera and the UVScope instrument, which is capable of counting individual photons in the range $300-650 \mathrm{~nm}$, with a time resolution of $10 \mathrm{~ns}$. The main result of this work is a strong correlation between the absolute NSB flux measured by UVscope and the fluctuations measured by the ASTRI-Horn camera that can be used as diagnostic tool to ensure the right behavior of the camera in view of the ASTRI-MiniArray implementation.
\end{abstract}

$3^{\text {th }}$ International Cosmic Ray Conference (ICRC 2021)

July 12th - 23rd, 2021

Online - Berlin, Germany

\footnotetext{
*Presenter
} 


\section{Introduction}

Imaging Atmospheric Cherenkov Telescopes (IACTs) opened a new window in the sky observation detecting the emission of cosmic gamma-ray sources at very high energies (VHE) through the Cherenkov light produced in the atmosphere by particle showers. The shower signal is embedded in the night sky background (NSB) whose intensity depends on geographical, environmental and atmospheric conditions [4].

As for all IACTs, the problem to correctly evaluate the NSB level is present even in the ASTRIHorn telescope ${ }^{1}$, so named in honor of the Italian astronomer Guido Horn D'Arturo, who pioneered the use of segmented mirrors in astronomy. The ASTRI-Horn telescope is located in Italy on Mount Etna, at the INAF "M.C. Fracastoro" observing station $\left(37.7^{\circ} \mathrm{N}, 15.0^{\circ} \mathrm{E}, 1740 \mathrm{~m}\right.$ a.s.1.); during the telescope commissioning phase, in December 2018 the Crab Nebula was detected above an energy threshold of $3 \mathrm{TeV}$ [5]. ASTRI-Horn is the prototype of nine similar telescopes forming the ASTRI-MiniArray (ASTRI-MA) that will be installed at the Teide Observatory, Canary Islands [1].

ASTRI-Horn is characterized by an optical system based on a dual-mirror Schwarzschild-Couder design and a camera at the focal plane composed of silicon photomultiplier (SiPM) sensors of $8 \times 8$ pixels, each of them with a sky-projected angular size of $0.19^{\circ}$ [2]. The SiPMs, read by a fast read-out electronics specifically designed [2, 12], are arranged in 21 detection units, named photon detection modules (PDMs), for a total effective field of view (FoV) of about $7.6^{\circ}$. The ASTRI-Horn focal plane is covered by a protective window and the entire camera, with its electronics and all ancillary devices, including a novel inner fiber-optic equipment (FOC) for relative calibration $[2,10]$ is closed in a basket whose top is open thanks to a lid system.

Several auxiliary instruments are present on site to monitor environmental and sky conditions; among them, UVscope [6, 7], a calibrated instrument devoted to the measurement of the NSB light in the wavelength range 300-650 $\mathrm{nm}$. The UVscope designed for ASTRI-Horn basically consists of: a photodetector with a time resolution of $10 \mathrm{~ns}$ equipped with its front-end and data acquisition electronics units working in single photon counting (SPC) mode, and an interface card for computer connection; a pinhole collimator to regulate the angular aperture of the detector and to protect its sensitive area against stray light; a motorized diaphragm to open/close the entrance pupil during night/day. The detector is a multi-anode photomultiplier tube (MAPMT) with its 64 anodes arranged in a matrix of $8 \times 8$ pixels, each of them with $0.55^{\circ}$ an angular aperture, equivalent to about $3 \times 3$ pixels of the ASTRI-Horn camera. The efficiency of the MAPMT detection unit was previously measured in the laboratory by comparison with a photodiode calibrated and certified by the National Institute of Standards and Technology (NIST). UVscope is mounted, co-aligned with the ASTRI-Horn camera axis, on the external structure of the telescope and it acquires data simultaneously with the ASTRI-Horn camera, without any interference with the main telescope data taking procedures. For the diffuse NSB evaluation, the UVscope pixels of the external frame are excluded because their content is influenced by effects on the edges; so the effective field of view of UVscope is about $3.5^{\circ}$. Being co-aligned with the ASTRI-Horn camera axis, the UVscope field of view covers the central area of $18 \times 18$ pixels of the ASTRI-Horn camera. The ASTRI-Horn camera is almost blind to the diffuse

${ }^{1}$ http://www.brera.inaf.it/astri-prototipo/ 
NSB but is able to detect the Poissonian fluctuations that it produces. So that, while the diffuse NSB level is directly evaluated by UVscope, in ASTRI-Horn it is derived by the $R M S$ produced at the random arrival of NSB photons. Such Poissonian noise is proportional to the diffuse NSB level and therefore can be correlated with measurements obtained by UVscope. Furthermore, in March 2019, the Variance method[11] was implemented in the ASTRI-Horn camera firmware: it allows to measure the NSB background and its variation by sampling the variance of each pixel at the not-occurrence of camera triggers.

In this contribution, we present the analysis of the diffuse NSB flux performed on real data acquired during the Crab Nebula observation campaigns between December 2018 and March 2019 and the resulting correlation between ASTRI-Horn and UVscope. A detailed description of the analysis and discussion of the results can be found in [3].

\section{ASTRI-Horn data reduction and analysis}

The analysis presented in this contribution is based on the images of triggered showers by the ASTRI-Horn camera. As they are expressed in term of ADC counts per pixel, the first step to be performed is their calibration and conversion to photo-electrons (p.e.). This requires the knowledge of the gain and pedestal of each pixel and, to this purpose, we made use of data obtained with the FOC system inner to the camera $[2,10]$ and of events acquired with the camera lids closed [3].

In the calibrated images, we considered only the ASTRI-Horn camera central region $(18 \times 18$ pixels) that matches the UVscope FoV. Background pixels in ASTRI-Horn images were identified applying a double cut cleaning procedure similar but complementary to the one used for the extraction of Cherenkov shower images, that allows us to discard all the fast signals (shower and muons) and to collect all the slow varying signal (diffuse NSB and stars) [5]. This procedure considers as background pixels the ones that have a signal lower than a first threshold $S 1$, or, if higher than $S 1$, without any neighbouring pixels with a signal higher than the second threshold $S 2$. We adopted the values $S 1=6$ p.e. and $S 2=12$ p.e. used by [9] to identify muon signals, that is also suitable to discard the showers being lower than the thresholds adopted for the analysis of Crab Nebula events [5].

From the Variance data, we know that ASTRI-Horn camera is able to detect stars with an increase of the $R M S$. The evaluation of the background then requires that pixels illuminated by stars must be excluded. The computation of the $R M S$ averaged over 5000 events, corresponding to an exposure of $\sim 2$ minutes, follows the two steps listed below:

1. identification of the pixels illuminated by stars: for each pixel of the camera central region, we computed the RMSs of the detected p.e. over 5000 events and accumulated the relative distribution. We then modeled the distribution with a Gaussian whose parameters (centroid and $\sigma$ ) were obtained by means of a fitting procedure. We considered as good pixels, i.e. not including stars, the ones whose $R M S$ is lower than $3 \sigma$.

2. evaluation of the average RMS: we computed a single p.e. distribution for the 5000 events and all good pixels ( $R M S$ lower than $3 \sigma$ ) and lying in the camera central region. It takes into account the statistics of the distribution as well as the systematics in the pixel gain calibration. We fitted 
the central part of this distribution with a Gaussian whose sigma is the $R M S$ that we correlate with the diffuse NSB flux as obtained by the UVscope data. Errors on the best-fit $R M S$ values are relative to $68 \%$ confidence level.

To cross-check the validity of this procedure, we used March 2019 observations when contemporary Variance data were also available. We were able to reproduce with our method the Variance values in each image as shown in Fig. 1 where some scientific data corresponding to the night of the March 6-7 2019 (left plot) are plotted together with the correspondent Variance data.
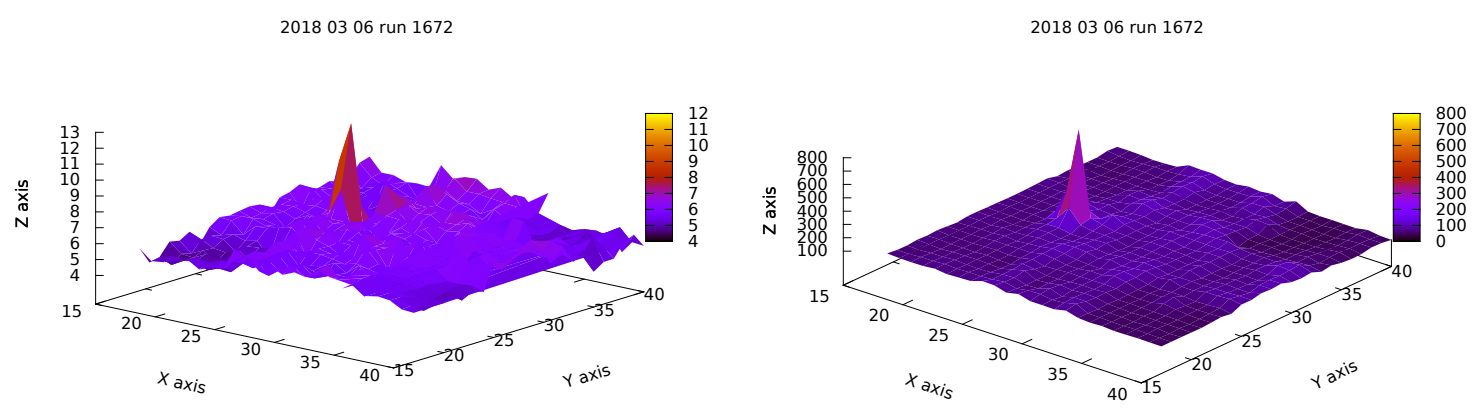

Figure 1: The left panel shows some scientific data corresponding to the night of the March 6-7 2019, for comparison the right panel plot the Variance data for the same night. The peak corresponds to Zeta Tauri, 1 deg off axis from the pointed source (Crab).

\section{Results}

Among all data we analysed, we found that only two nights (2018 December 7-8 and 2019 March 6-7) were with clear or a bit cloudy sky therefore suitable for the correlation with UVscope. The temporal evolution of the $R M S$, computed for the two selected nights and compared with the flux measured by UVscope is shown in Figure 2 where the light curves relative to the night 2018 December 7-8 and 2019 March 6-7 are displayed in the left and right plots, respectively. In each plot, we report the $R M S$ data (top panels) measured by ASTRI-Horn every $\sim 2$ minutes and of the NSB flux measured by UVscope every second (bottom panels) since the beginning of the observation.

We note that there is an overall agreement between the two instrument measurements. In particular, it is evident that the RMS values measured by ASTRI-Horn follow the jumps in the NSB flux detected when the telescope moves to different observing directions (see vertical dashed lines in the plots) because of the on-off pointing procedure applied during the Crab Nebula observation.

However, ASTRI-Horn RMSs do not vary significantly from the beginning up to $0.2 \mathrm{~h}$ in December 7-8 night, when the telescope was pointing towards Catania city, at variance with the steepening after 7.5h due to the Moon and Sun rise, when the ASTRI-Horn points better correlate with UVscope. The small increase in UVscope data at $4 \mathrm{~h}$ after the beginning of March 6-7 observation is due to clouds not detectable by ASTRI-Horn. 

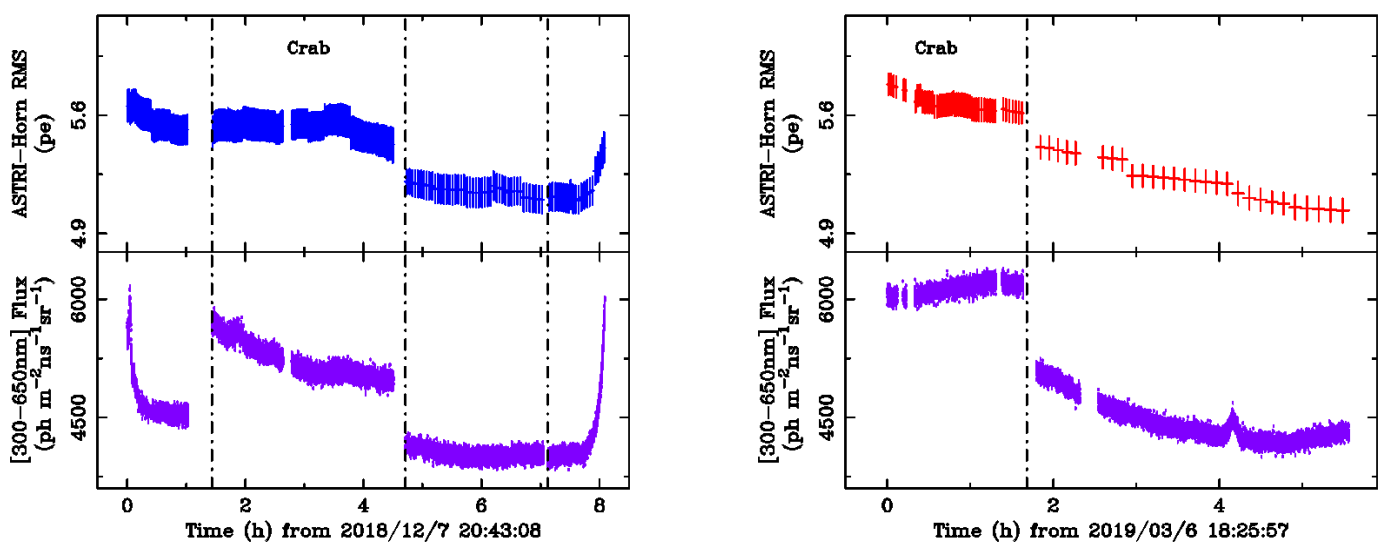

Figure 2: Temporal evolution of the $R M S$ s measured by ASTRI-Horn (top panels) and the NSB fluxes measured by UVscope every second (bottom panels) relative to the observing night 2018 December 7-8 (left plot) and 2019 March 6-7 (right plot). The vertical dashed lines indicate variations of the telescope pointings.

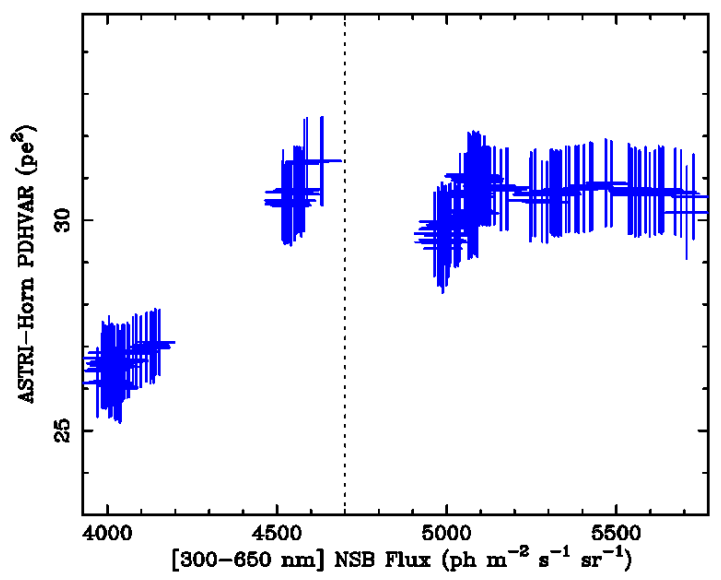

Figure 3: ASTRI-Horn PDHVAR - UVscope fluxes correlation for the 7-8 December 2018 night. The dashed black vertical line indicates the level we used to distinguish the linear regime from saturation.

The behaviour of ASTRI-Horn camera with respect to the NSB variations was better investigated with the correlation with UVscope measurements. To this purpose, it was necessary to average the UVscope flux over the ASTRI-Horn time intervals. In fact, UVscope integrates the sky signals every second, while the ASTRI-Horn RMSs are averaged over $\sim 2$ minutes.

Moreover, considering that the variance of the NSB fluctuations is proportional to the flux (see Sect. 4), we decided to correlate the variance $R M S^{2}=P H D V A R$ derived with our analysis with the UV scope measurements. Plotting PHDVAR as a function of the NSB flux measured by UVscope for the two considered observation nights, we note that, in both nights, the PHDVARs vary linearly with 
the NSB flux at low flux level, while, at higher fluxes, the PHDVARs seem to reach a saturation level. As an example, Figure 3 shows the data relative to the 7-8 December night.

\section{Discussion}

The RMS of the background fluctuation in the ASTRI-Horn camera derives from mainly two contributions: the intrinsic standard deviation of the electronics plus the detector noise observed in dark condition and the standard deviation induced by the NSB level that, being the signal Poissonian, is linearly correlated to the flux $\left(A \times F l u x_{N S B}\right)$. The correlation factor $A$ depends on several ingredients, as the two instrument working ranges, the ASTRI-Horn total efficiency (optics reflectivity plus filter transmission and SiPM photo-detection efficiency) and the camera electronics operation mode (i.e. the peak detector). Its value is not expected to vary unless one of the characteristics of the two systems changes. The linear correlation between UVscope flux and ASTRI-Horn variance observed when the diffuse NSB flux is below $\sim 4700 \mathrm{ph} \mathrm{m}^{-2} \mathrm{~ns}^{-1} \mathrm{sr}^{-1}$ (see Fig. 3), is then in agreement with theory, while, above this flux, the camera saturate. Laboratory checks and ah-hoc simulations showed that the cause of this flattening is a current consumption limit in the power system of all the camera pixels [3].

We analysed the linear regime in the two considered nights that allowed us to obtain statistically significant values for the correlation. Fig. 4 shows these data together with the relative best fitting lines.

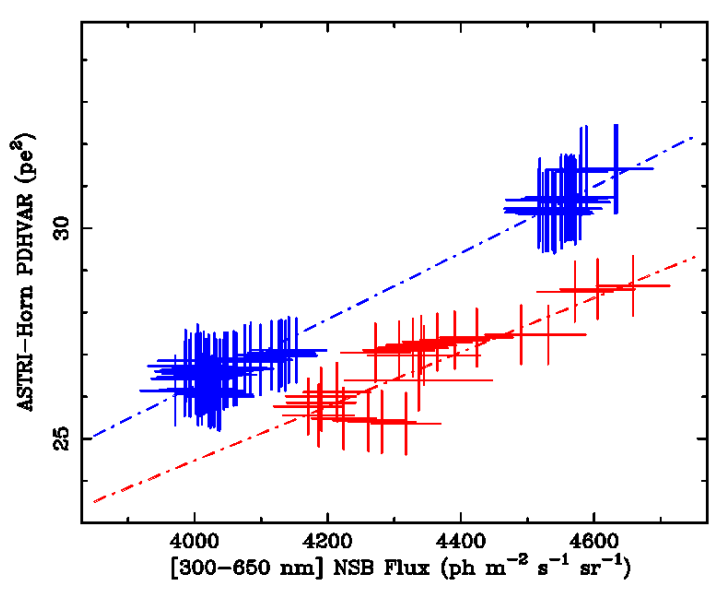

Figure 4: ASTRI-Horn PHDVARs as function of the UVscope fluxes for the 7-8 December 2018 (blue) and for the 6-7 March 2019 (red) night. The dash-dotted lines indicate the best fit models.

The values of the correction factor A measured on December 2018 and on March 2019 are not compatible, being their ratio $0.77 \pm 0.06$. However, we know, from the results obtained with the muon ring analysis [9], that the telescope effective area measured in March 2019 was degraded by 25\% after a strong Etna eruption in February 2019. The lower correlation slope can then be ascribed to the reduction of the optical throughput. This results makes the correlation between the ASTRI-Horn 
variance and the UVscope diffuse NSB flux evaluation a good parameter for checking the stability of the telescope optical throughput together with other calibration methods (i.e. muon ring analysis, external calibrated light [8]).

\section{Conclusion}

In this contribution we investigated the correlation between the NSB fluxes measured by UVscope and ASTRI-Horn background RMSs using contemporary data collected during the Crab Nebula observation campaign performed between December 2018 and March 2019. We found that only two sets of data were suitable for this analysis having long enough observation intervals with a NSB level lower than $\sim 4700 \mathrm{ph} \mathrm{m}^{-2} \mathrm{~ns}^{-1} \mathrm{sr}^{-1}$ above which the camera response starts to saturate because of a limit in the power supply. We discovered that there is a linear correlation between the Variance measured in the ASTRI-Horn camera pixels and the flux measured by UVscope, quantified through the slope $A$. This correlation that depends on several parameters is expected not to vary unless some of the two instruments characteristic change. We detected a reduction of $A$ of about $25 \%$ between the two datasets due to a degradation of the optics reflectivity. This observation suggests that the study of the correlation between the Variance measured by ASTRI-Horn telescope and the NSB flux measured by UVscope is an useful diagnostic tool that can quickly point out reduction of the overall throughput.

The limit in the PDM power system of ASTRI-Horn, that causes the saturation effect, has been increased in the updated version of the camera to greatly alleviate the saturation problem. Considering that UVscope will continue to be operative during the future observation campaigns, the results presented in this contribution could be consolidated with larger data set. Moreover, the saturation effect will not be present in the cameras designed for the ASTRI-MA telescopes where an upgraded version of the power system will be able to supply a constant power up to 10 times the current level. At the same time, a new generation of UVscope, called UVSiPM, equipped with SiPM sensors instead of the MAPMT, to better match the spectral response of the ASTRI camera, is under design for its use in ASTRI-MA.

\section{Acknowledgement}

This work was conducted in the context of the ASTRI Project. It is supported by the Italian Ministry of University and Research (MUR) with funds specifically assigned to the Italian National Institute for Astrophysics (INAF). We acknowledge support from the Brazilian Funding Agency FAPESP (Grant 2013/10559-5) and from the South African Department of Science and Technology through Funding Agreement 0227/2014 for the South African Gamma-Ray Astronomy Programme. This work has been supported by H2020-ASTERICS, a project funded by the European Commission Framework Programme Horizon 2020 Research and Innovation action under grant agreement n. 653477. IAC is supported by the Spanish Ministry of Science and Innovation (MICIU). Authors would like to thank Filippo Ambrosino for his insightful comments. 


\section{References}

[1] Antonelli L (2021) The ASTRI mini-array at Teide Observatory. In: 37th International Cosmic Ray Conference (ICRC2021), International Cosmic Ray Conference Series, vol This Proceeding

[2] Catalano O, Capalbi M, Gargano C, et al. (2018) The ASTRI camera for the Cherenkov Telescope Array. In: , Society of Photo-Optical Instrumentation Engineers (SPIE) Conference Series, vol 10702, p 1070237, 10.1117/12.2314984

[3] Compagnino AA, Mineo T, Maccarone MC, et al. (2021) The ASTRI-Horn telescope: comparison with the auxiliary UVscope measurements as a feasible diagnostic and calibration tool. Submitted to Experimental Astronomy

[4] Leinert C, Bowyer S, Haikala LK, et al. (1998) The 1997 reference of diffuse night sky brightness. 127:1-99, 10.1051/aas:1998105

[5] Lombardi S, Catalano O, Scuderi S, et al. (2020) First detection of the Crab Nebula at TeV energies with a Cherenkov telescope in a dual-mirror Schwarzschild-Couder configuration: the ASTRI-Horn telescope. 634:A22, 10.1051/0004-6361/201936791, 1909.12149

[6] Maccarone MC, Catalano O, Giarrusso S, et al. (2011) Performance and applications of the UVscope instrument. Nuclear Instruments and Methods in Physics Research A 659:569-578, 10.1016/j.nima.2011.08.004

[7] Maccarone MC, La Rosa G, Catalano O, et al. (2021) UVscope and its application aboard the ASTRI-Horn telescope. Experimental Astronomy 10.1007/s10686-021-09728-6, 2103.02233

[8] Mineo T., Maccarone M.C., Antonelli L.A., et al. (2021) Tools and Procedures for the ASTRI Mini-Array Calibration. In: 37th International Cosmic Ray Conference (ICRC2021), International Cosmic Ray Conference Series, vol This Proceeding

[9] Mineo T, Maccarone MC, Compagnino A, et al. (2019) Muon calibration of the ASTRI-Horn telescope: preliminary results. In: 36th International Cosmic Ray Conference (ICRC2019), International Cosmic Ray Conference, vol 36, p 744, 1907.09197

[10] Rodeghiero G, Catalano O, Segreto A, et al. (2014) Illumination technique for the relative calibration of the ASTRI SST-2M camera. Nuclear Instruments and Methods in Physics Research A 764:176-185, 10.1016/j.nima.2014.07.038

[11] Segreto A, Catalano O, Maccarone MC, et al. (2019) Calibration and monitoring of the ASTRIHorn telescope by using the night-sky background measured by the photon-statistics ("variance") method. In: 36th International Cosmic Ray Conference (ICRC2019), International Cosmic Ray Conference, vol 36, p 791, 1909.08750

[12] Sottile G, Catalano O, La Rosa G, et al. (2016) ASTRI SST-2M camera electronics, Society of Photo-Optical Instrumentation Engineers (SPIE) Conference Series, vol 9906, p 99063D. $10.1117 / 12.2232464$ 